\title{
Development and Validation of a Diabetic Retinopathy Screening Modality Using a Hand-Held Nonmydriatic Digital Retinal Camera by Physician Graders at a Tertiary-Level Medical Clinic: Protocol for a Validation Study
}

Mapa Mudiyanselage Prabhath Nishantha Piyasena ${ }^{1}$, MSc, MBBS; Venkata S Murthy Gudlavalleti ${ }^{1}$, MD, MSc; Clare Gilbert $^{1}$, MD, FRCOphth, MSc; Jennifer LY Yip ${ }^{1}$, MRCOphth, FFPH, PhD; Tunde Peto ${ }^{2}$, MD, MHealth Ed, PhD, FHCO; David MacLeod ${ }^{1}$, PhD; Charith Fonseka ${ }^{3}$, MS, FRCS, FRCOphth; Aruna Kulatunga ${ }^{4}$, MD, FRCP; BGWMKCB Bandutilake $^{3}$, MD, FRCOphth; Mangala Dhanapala ${ }^{3}$, MD, FICO; Lalani Pathirana ${ }^{4}$, MBBS, DFM; Heshani Dissanayake ${ }^{4}$, MBBS

${ }^{1}$ Clinical Research Department, International Centre for Eye Health, London School of Hygiene and Tropical Medicine, London, United Kingdom

${ }^{2}$ School of Medicine, Dentistry and Bio-medical Science, Queen's University, Belfast, Ireland

${ }^{3}$ Vitreo-retina Unit, National Eye Hospital, Colombo, Sri Lanka

${ }^{4}$ National Hospital of Sri Lanka, Colombo, Sri Lanka

Corresponding Author:

Mapa Mudiyanselage Prabhath Nishantha Piyasena, MSc, MBBS

Clinical Research Department

International Centre for Eye Health

London School of Hygiene and Tropical Medicine

Keppel Street

London, WC1E 7HT

United Kingdom

Phone: 442079588316

Fax: 442074365389

Email: Prabhath.piyasena@1shtm.ac.uk

\section{Abstract}

Background: Visual impairment and blindness from diabetic retinopathy (DR), which can be reduced by early screening and treatment, is an emerging public health concern in low-income and middle-income countries (LMICs) owing to the increasing prevalence of diabetes mellitus (DM). However, no systematic screening exists in most LMIC settings. The Western province of Sri Lanka has the highest prevalence of DM (18.6\%) in the country. A situational analysis identified a marked gap in DR screening (DRS) and treatment services uptake in this region; only opportunistic screening is practiced currently.

Objective: The aim of this protocol is to describe the methods of development and validation of a DRS intervention using a hand-held nonmydriatic digital camera by physician graders in a non-ophthalmological setting at a tertiary-level medical clinic to propose a valid and feasible modality to improve uptake.

Methods: DRS modality was developed after assessing barriers and identifying the most appropriate personnel, methods, and location for screening services, following formative research work. The validation will be conducted in a public sector tertiary care center in the Western province of Sri Lanka. The selected physicians will be trained on capturing and grading images according to a valid locally adopted protocol. Two physicians rated high on training will screen a sample of 506 people with DM at a medical clinic. They will use nonmydriatic and mydriatic 2-field imaging strategy. The validity of the proposed screening procedure will be assessed and compared with the mydriatic indirect biomicroscopic examination by a senior retinologist.

Results: The validity of screening by physician graders will be analyzed and the sensitivity, specificity, and predictive values (with 95\% CIs) calculated by the dilation status and for each grader. The diagnostic accuracy at each level of severity of DR will be assessed to define the most appropriate referable criteria. Data is currently being collected.

Conclusions: The outcome of this study will be useful for the detection of a defined level of DR at non-ophthalmological setting to filter the people with DM before referral to an eye clinic. This will be helpful to improve the uptake and identify risk groups 
in advance to prevent sight-threatening DR. Furthermore, evidence from this study will be useful for the implementation of a DRS program in this region and in similar communities.

International Registered Report Identifier (IRRID): PRR1-10.2196/10900

(JMIR Res Protoc 2018;7(12):e10900) doi: 10.2196/10900

\section{KEYWORDS}

diabetes; diabetic retinopathy; digital imaging; hand-held camera; mydriatic; nonmydriatic; physician grader; screening; Sri Lanka

\section{Introduction}

The prevalence of diabetes mellitus (DM) and the number of people affected by DM is increasing rapidly in all regions. The International Diabetes Federation estimated that 425 million people had diabetes in 2017, which will increase to 629 million in 2045 globally [1]. This increase is expected to be the highest in low-income and middle-income countries (LMICs) compared with high-income countries (HIC) [2]. Diabetic retinopathy (DR) is a common microvascular complication of DM, which can lead to visual impairment and blindness if not detected early and treated [3]. Many studies have reported that visual loss from DR can be largely prevented by early screening and appropriate treatment [4-6]. Diabetic retinopathy screening (DRS) can be done in 2 ways, systematic screening similar to national-level programs in HIC versus opportunistic screening and case detection, which is common in low-income settings. Most LMICs are unlikely to have full population-based screening program owing to resources constraints. The current method of DRS in most LMICs is direct ophthalmoscopy, which has a lower diagnostic accuracy and found to be ineffective even after training [7]. The mydriatic biomicroscopic examination by an ophthalmologist is practically not possible in these countries owing to a low number of ophthalmologists, and eye clinics are overburdened with highly prevalent blinding conditions such as cataract [8].

The reasons for the unavailability of DRS programs (DRSPs) in LMIC settings are mostly attributed to the lack of skilled human resources, financial resources, and evidence of what works in the local system [9-11]. Therefore, it would be important to understand the approaches for screening, especially in non-ophthalmological settings. Conventional digital cameras need a larger space, skilled photographers, and large image storage devices. In addition, systematic screening using sophisticated table-top imaging systems incur high capital investment though they are cost-effective [12]. Hand-held digital cameras are easy to move, require minimum space, minimum power consumption, and are user-friendly [13]. In addition, nonmydriatic hand-held cameras are less discomforting to participants and can be used while people with DM are waiting in front of a physician for consultation. The usage of a camera without pupil dilatation is comfortable to people with DM, as well as easy for providers. However, the latter depends on the quality of the image available for grading [14].

Various photographic studies have looked at the diagnostic test accuracy of DRS using digital imaging. Most of these studies used static table-top imaging systems and were conducted in HICs. These studies have shown a sensitivity of $68 \%-97 \%$ and a specificity of $71 \%-100 \%$ in nonmydriatic imaging using ophthalmic human resources as index graders [15-18]. Similarly, in mydriatic imaging, most of the studies have used table-top imaging systems, ophthalmic human resources as index test graders, and were conducted in HICs. These studies have shown a sensitivity of $77 \%-97 \%$ and a specificity of $76 \%-98 \%$ in mydriatic digital imaging [19-22]. There is a gap in evidence in digital retinal imaging in LMICs using non-ophthalmic human resources. In addition, the usage of context-specific imaging systems, such as hand-held digital retinal camera, in non-ophthalmic setting was not reported in the current literature.

Sri Lanka has achieved remarkable development in the health sector; however, there are public health concerns such as DR which have not been addressed to date [23]. The crude prevalence of DM in Sri Lanka was $12.6 \%$ (>20 years), being highest in the Western province $(18.6 \%, 95 \%$ CI $15.8 \%-21.5 \%)$ [24]. In the Western province, there are approximately 750,000 (age $>18$ years) people with DM, 20\% $(150,000 / 750,000)$ of whom are likely to have nonproliferative DR (NPDR). A situational analysis conducted in this region has shown that the number of people undergoing opportunistic screening and free treatment in the public sector was far lower than the estimated need [25]. There is no systematic DRS in the Western province despite the high prevalence of DM [25]. In addition, there is no published data on this topic from Sri Lanka. The aim of this protocol is to describe the methods of validation of a DRS approach using digital imaging by physician graders in a tertiary-level public sector medical clinic. This study will demonstrate the functional and technical feasibility of using a hand-held digital camera in an LMIC non-ophthalmological setting and assess the diagnostic accuracy.

\section{Methods}

\section{Ethics Approval}

Ethics review committees of National Eye Hospital (Colombo, Sri Lanka) and London School of Hygiene \& Tropical Medicine (United Kingdom) granted ethics approval.

\section{Development of the Diabetic Retinopathy Screening Modality and Training}

The initial formative research showed that nonmydriatic digital retinal imaging at medical clinics by general physicians was a potential option for the local setting. We selected 9 general physicians from a tertiary-level institution following informed consent, and they underwent a competency-based training by 2 retinologists from a tertiary center, which included the following: capturing retinal fields using a hand-held fundus camera, identification of signs of DR (including macular signs) using images, and DR grading according to an adapted 
classification system (Table 1). DR signs are graded at 4 levels as follows: none $=\mathrm{R} 0$, mild NPDR $=\mathrm{R} 1$, moderate $\mathrm{NPDR}=\mathrm{R} 2$, severe $\mathrm{NPDR}=\mathrm{R} 3$, and proliferative $\mathrm{DR}$ and above $=\mathrm{R} 4$. Macular changes are graded as follows: none $=\mathrm{M} 0$ (maculopathy absent) and exudate(s) or blot hemorrhage(s) within 2-disc diameters from the center of the fovea=M1 (maculopathy present). Guidelines were used to standardize reporting of image quality, which included ungradable images based on the proportion of the retina visible for grading (Figure 1). After the training, physicians were tested using a set of standard images of DR, and the two physicians who reached the required level of agreement with the retinologists $(\kappa=.8-.9)$ were selected as graders in the validation study.

Table 1. Adapted diabetic retinopathy classification for the validation study.

\begin{tabular}{|c|c|c|c|c|c|}
\hline Signs & No $\mathrm{DR}^{\mathrm{a}}(\mathrm{R} 0)$ & $\begin{array}{l}\text { Mild } \mathrm{BDR}^{\mathrm{b}} \text { or } \\
\mathrm{NPDR}^{\mathrm{c}}(\mathrm{R} 1)\end{array}$ & $\begin{array}{l}\text { Moderate BDR or } \\
\text { NPDR (R2) }\end{array}$ & Severe NPDR (R3) & $\operatorname{PDR}^{\mathrm{d}}(\mathrm{R} 4)$ \\
\hline Microaneurysms & No & Few & Multiple & Multiple & Present \\
\hline Hard exudates ${ }^{\mathrm{e}}$ & No & Few & Multiple & Multiple & Present \\
\hline Cotton wool spots & No & Occasional & Multiple & Multiple & Present \\
\hline Intraretinal hemorrhage $\mathrm{e}^{\mathrm{e}}$ & No & Few & $>20$ in $1-3$ quadrants & $>20$ in 4 quadrants & Present \\
\hline Venous beading & No & Occasional & $\begin{array}{l}\text { Present in 1-2 quad- } \\
\text { rants }\end{array}$ & $\begin{array}{l}\text { Present in }>2 \text { quad- } \\
\text { rants }\end{array}$ & Present \\
\hline IRMA $^{\mathrm{f}}$ & No & No & Present $\sim 1$ quadrant & $\begin{array}{l}\text { Prominent }>1 \text { quad- } \\
\text { rant }\end{array}$ & Present \\
\hline $\mathrm{NVD}^{\mathrm{g}}$ & No & No & No & No & Present \\
\hline $\mathrm{NVE}^{\mathrm{h}}$ & No & No & No & No & Present \\
\hline Vitreous or preretinal hemorrhage & No & No & No & No & $\begin{array}{l}\text { Present-advanced } \\
\text { PDR }\end{array}$ \\
\hline Traction & No & No & No & No & $\begin{array}{l}\text { Present-advanced } \\
\text { PDR }\end{array}$ \\
\hline Fibrosis & No & No & No & No & $\begin{array}{l}\text { Present-advanced } \\
\text { PDR }\end{array}$ \\
\hline
\end{tabular}

${ }^{\mathrm{a} D R}$ : diabetic retinopathy.

${ }^{\mathrm{b}}$ BDR: Background DR.

${ }^{\mathrm{c}}$ NPDR: nonproliferative DR.

${ }^{\mathrm{d}}$ PDR: proliferative DR.

${ }^{\mathrm{e}}$ Not within the definition of maculopathy.

${ }^{f}$ IRMA: Intraretinal microvascular abnormalities.

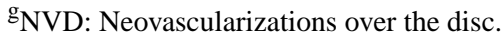

$\mathrm{h}_{\text {NVE: Neovascularizations elsewhere. }}$

Figure 1. Evaluation of image quality-levels of gradeability based on the proportion of the image that can be graded.

\begin{tabular}{|c|c|c|c|}
\hline \multicolumn{2}{|c|}{ Gradable } & Ungradable \\
\hline $100 \%$ Gradable & $75 \%$ Gradable & $50 \%$ Gradable & $<50 \%$ Visible \\
\hline 6 & & & \\
\hline
\end{tabular}




\section{Sample Calculation and Recruitment}

The sample size $(n=405)$ was calculated on the basis of $95 \%$ CIs, $10 \%$ margin of error, expected sensitivity $70 \%$, and the prevalence of moderate NPDR among people with DM of $20 \%$. Then, we inflated the sample size by an additional $25 \%(\mathrm{n}=101$, total $n=506$ ) to take account of ungradable images. An interim analysis will be undertaken to ascertain the level of ungradable images (ie, $<50 \%$ of the retina visible) and the sample size increased, if required.

This study is a prospective observational study by design. A consecutive sample of diagnosed people with DM (age >18 years) without previous DRS at an eye clinic will be eligible to participate, after giving written informed consent. Eligible participants will be recruited by trained research assistants when people with DM present for routine medical care at the main tertiary center in Colombo. People with DM with previous retinal screening, DR-related treatment (laser treatment, intravitreal injections, and pars plana vitrectomy), and those who were currently under any DRSP or treatment will be excluded from the study. Figure 2 shows the participants' flow diagram. Participants' characteristics will be documented in a questionnaire schedule by research assistants on recruitment.

Figure 2. Participants' flow diagram in the validation.

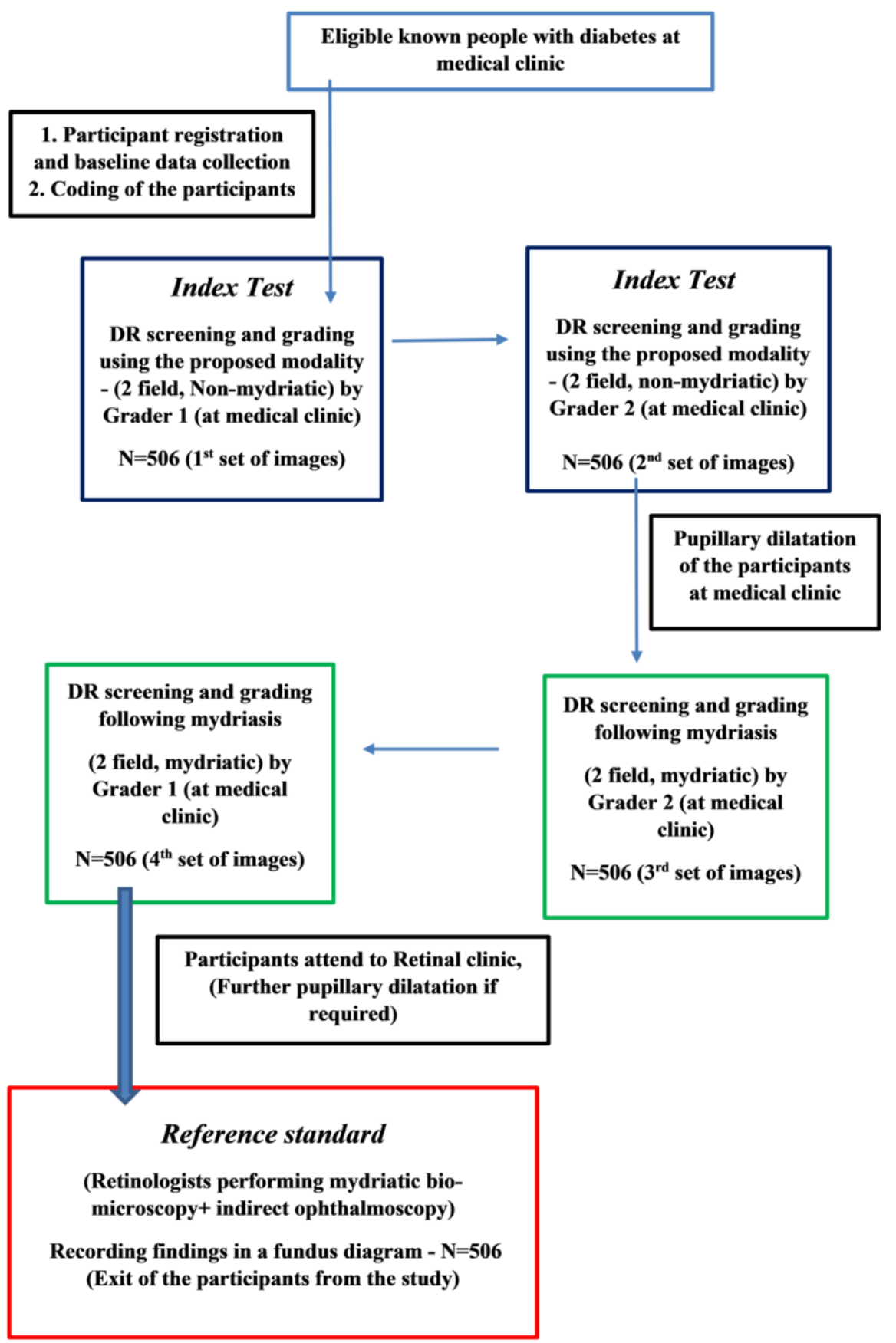


Figure 3. Two retinal fields captured.

\begin{tabular}{|l|l|}
\hline Field 1. Centered on the macula & Field 2. Centered on the optic disc \\
\hline & \\
\hline OD
\end{tabular}

\section{Imaging System, Capturing Images, and Grading}

Two-field nonmydriatic and mydriatic retinal images will be captured and stored (Figure 3). Participants will undergo digital retinal imaging (using VISUSCOUT100 hand-held nonmydriatic fundus camera-2017; Carl Zeiss, Germany) by the physician graders at the time of presentation. This imaging system has a $40^{\circ}$ field-of-view with 5 megapixels (type of camera sensor: complementary metal-oxide semiconductor; resolution $800 \times 480$ ) and captures color and red-free images in a focus range of -20 $\mathrm{D}$ (diopters) to $+20 \mathrm{D}$. The minimum pupil size required is 3.5 $\mathrm{mm}$, and 9 light-emitting diodes are available for internal fixation.

First, 2-field (first field-macula centered, second field-disc centered; Figure 3 ), $40^{\circ}$ retinal images will be captured in each eye by each physician grader without pupillary dilatation. Subsequently, participants' pupils will be dilated using $2.5 \%$ phenylephrine, and the same fields will be captured, following adequate mydriasis $(5-6 \mathrm{~mm})$.

Each set of images will be coded and stored by research assistants after capturing. The coded image sets will be given back to the same physician graders for grading. During grading, nonmydriatic images will be graded first. The graders will be masked to the history and clinical examination findings. The retinopathy and macular signs will be identified and entered by physician graders into a hardcopy data table. Finally, it will be entered into a Microsoft Excel datasheet by research assistants. The grading data consistency checks and cleaning will be done by an independent statistician.

\section{Reference Test}

The reference test will entail a detailed, dilated fundus examination by an experienced retinologist using slit-lamp biomicroscopy with a 90D lens and indirect ophthalmoscopy using a 20D lens. After imaging, this examination will take place as early as possible. The retinologist will be masked to the clinical status and physician graders' findings. In addition, a detailed anterior segment examination (clarity of cornea and status of the lens) and media (vitreous) examination will be done by the reference test grader. The lens opacity will be graded according to the lens opacity classification system, version 111.

\section{Quality Assurance and Agreement Analysis}

For quality assurance, $15 \%$ of each nonmydriatic and mydriatic image sets will be evaluated by the retinologist for technique, ability to image the required field, and gradeability. Then, $15 \%$ of each hundred image sets will be given back to physician graders for double grading to assess the repeatability and intragrader agreement in the first and second attempts of grading images. A sample of the same image sets $(n=200)$ will be graded by the retinologist to calculate the intergrader agreement.

\section{Data Analysis}

We will analyze the validity of screening by physician graders and calculate the sensitivity, specificity, and predictive values with $95 \%$ CIs for each method of screening and by the grader. The analysis will be conducted by including and excluding ungradable images and considering each eye as a unit of analysis and by a person considering the worst eye. Intragrader and intergrader agreement (kappa) for both mydriatic and nonmydriatic index tests will be calculated and compared with the findings by the retinologist. A subgroup analysis will be conducted for the identification of the presence or absence of DR (any DR), moderate NPDR, and above with or without macular signs to make recommendations for a referable criterion for the local context in DRS by physician graders.

\section{Results}

The physician graders have been trained, and currently, validation is being done in the Western province of Sri Lanka. The results of this study will be published in detail according to the Quality Assessment of Diagnostic Accuracy Study guidelines [26]. Data will be entered using a Microsoft Excel (2016) worksheet and transferred into STATA/IC-v14.2 analytical package following cleaning, consistency checks, and analysis. The sensitivity, specificity, and predictive values for 
each strategy and each level of DR will be presented using the same variables of 2 physician graders (nonmydriatic and mydriatic separately) compared with the reference standard, along with $95 \%$ CIs.

\section{Discussion}

The level of skills acquired by physician graders is an important factor in the screening outcome. Different non-ophthalmologist graders have successfully conducted DRS in some settings [27-29]. We will describe the diagnostic accuracy of the detection of DR by physician graders. In addition, we will be able to study the effect of a range of population characteristics on the validity of detecting DR using imaging and understand the role of non-ophthalmic personnel to make recommendations for a systematic DRSP. In addition, we will describe the referral criterion applicable to this local context based on the validation study results. Defining a referable level DR at a non-ophthalmological setting, in a context where there is no systematic DRS, will filter out those not needing a referral and therefore reduce the workload at an ophthalmologist's clinic. The 7-field imaging strategy used in early treatment diabetic retinopathy study is considered as the gold standard in DRS [30]. However, this technique is practically not feasible in this context owing to resources constraints. Therefore, we proposed to use the locally accepted reference standard of retinologists' examination as the suitable reference standard. Digital retinal imaging has previously shown diagnostic accuracy levels that would comply with the accepted standards of established national-level screening programs [15,22,31].

A few studies (conducted in HICs) have used non-ophthalmologist human resources in DRS, with which we could compare our results. In Singapore, a nonmydriatic fundus camera showed a sensitivity of $69.8 \%$ (95\% CI $61.3 \%-77.2 \%$ ) and a specificity of $94.4 \%$ (95\% CI $92.3 \%-96.1 \%)$ for nonphysician graders using a single field [32]. A study in the
United Kingdom on DRS by general practitioners using 35-mm color images showed that detecting any level of DR increased from $62.6 \%$ (95\% CI 55.9\%-69.4\%) with direct ophthalmoscopy to $79.2 \%$ (95\% CI $73.6 \%-84.9 \%$ ) using retinal photographs, and specificity remained unchanged (direct ophthalmoscopy $75.0 \% \quad[95 \%$ CI $\quad 69.5 \%-80.5 \%]$ vs $73.5 \% \quad[95 \%$ CI $68.0 \%-79.1 \%$ ]) [33]. They concluded that retinal photography by trained general practitioners in primary care settings could attain an acceptable level of detection of sight-threatening DR (87\%) [33]. In Thailand, the use of single-field digital nonmydriatic imaging showed a sensitivity of $80 \%$ and a specificity of $96 \%$ in a sample of people with DM, where $54.7 \%$ people with DM were aged 41-60 years and $45.3 \%$ people with DM had diabetes since 1-5 years [34].

Another important consideration in this study would be the gradeability of images. The image gradeability will depend on the lens opacity, media opacity, pupil size, and reflectivity of the fundus. We envisaged poor gradeability in nonmydriatic imaging considering the high prevalence of cataract in this local setting. Furthermore, iris color, age, and other population characteristics may affect the quality of images [14]. Scanlon et al showed that in the $>80$-years age group, the technical failure rates reduced from $41.6 \%$ to $16.9 \%$ following mydriasis. This study concluded that the odds of having one eye ungradable increases by $2.6 \%$ (95\% CI $1.6 \%-3.7 \%)$ for each extra year since the diagnosis of DM and major cause of ungradability was having central cataract $(57 \%)$ [35]. We will describe the factors affecting gradeability of images in addition to the diagnostic test accuracy results.

In this study, we will demonstrate the diagnostic accuracy of physician graders compared with the retinologist to make recommendations for developing an integrated DRSP in LMICs where there is no systematic DRS. The outcome of this study will be useful for the implementation of a systematic DRSP in this region and similar communities.

\section{Acknowledgments}

Investigators thank the Association of Vitreo Retina Specialists of Sri Lanka for the logistical and operational support provided in conducting this study. We also have a research degree student project grant awarded to MMPNP from Queen Elizabeth Diamond Jubilee Trust, United Kingdom, coordinated through Commonwealth Eye Consortium, UK.

\section{Authors' Contributions}

MMPNP conceived the project idea. MMPNP is coordinating and conducting this research project in the Western province as a fulfillment of a research degree. GVSM and JLYY supervised the student work. GVSM, CG, JLYY, TP, and DM made substantial contributions for the concept development, methodology, and study design. CF is the Principal Investigator of the project from collaborating institution and supervised the project work locally. AK will supervise the project-related work of DRS at the medical unit. MD and KB conducted the training of physician graders and KB will conduct the reference standard clinical examination of participants. $\mathrm{MD}$ and $\mathrm{KB}$ will involve in managing those study participants who require further investigations and treatment. LP and HD will conduct the validation of DRS intervention at present in the Western province.

\section{Conflicts of Interest}

None declared.

\section{References}

1. IDF. International Diabetes Federation. International Diabetes Federation-Diabetes Atlas - 08th Edition - 2017 URL: https:/ /www.idf.org/e-library/epidemiology-research/diabetes-atlas.html [accessed 2018-07-05] [WebCite Cache ID 70gZUlK5J] 
2. Shaw JE, Sicree RA, Zimmet PZ. Global estimates of the prevalence of diabetes for 2010 and 2030. Diabetes Res Clin Pract 2010 Jan;87(1):4-14. [doi: 10.1016/j.diabres.2009.10.007] [Medline: 19896746]

3. Williams R, Airey M, Baxter H, Forrester J, Kennedy-Martin T, Girach A. Epidemiology of diabetic retinopathy and macular oedema: a systematic review. Eye (Lond) 2004 Oct;18(10):963-983. [doi: 10.1038/sj.eye.6701476] [Medline: $\underline{15232600}$ ]

4. Intensive blood-glucose control with sulphonylureas or insulin compared with conventional treatment and risk of complications in patients with type 2 diabetes (UKPDS 33). UK Prospective Diabetes Study (UKPDS) Group. Lancet 1998 Sep 12;352(9131):837-853. [Medline: $\underline{\text { 9742976] }}$

5. Klein R, Knudtson MD, Lee KE, Gangnon R, Klein BEK. The Wisconsin Epidemiologic Study of Diabetic Retinopathy: XXII the twenty-five-year progression of retinopathy in persons with type 1 diabetes. Ophthalmology 2008

Nov;115(11):1859-1868 [FREE Full text] [doi: 10.1016/j.ophtha.2008.08.023] [Medline: 19068374]

6. The Diabetic Retinopathy Study Research Group. Indications for photocoagulation treatment of diabetic retinopathy: Diabetic Retinopathy Study Report no. 14.. Int Ophthalmol Clin 1987;27(4):239-253. [Medline: 2447027]

7. Gerhs K, Chong L, Gusman G, Street D. Can we educate primary care physicians about diabetic retinopathy after graduation? Preliminary results of the diabetic retinopathy education study. Invest Ophthalmol Vis Sci 1993;34:5.

8. Bamashmus MA, Gunaid AA, Khandekar R. Regular visits to a diabetes clinic were associated with lower magnitude of visual disability and diabetic retinopathy-a hospital-based historical cohort study in yemen. Diabetes Technol Ther 2009;11(1):45-50. [doi: 10.1089/dia.2007.0299] [Medline: 19132855]

9. Adriono G, Wang D, Octavianus C, Congdon N. Use of eye care services among diabetic patients in urban Indonesia. Arch Ophthalmol 2011 Jul;129(7):930-935. [doi: 10.1001/archophthalmol.2011.147] [Medline: 21746983]

10. Burgess PI, Msukwa G, Beare NAV. Diabetic retinopathy in Sub-Saharan Africa: meeting the challenges of an epidemic. BMC Medicine 2013;11(1) [FREE Full text]

11. Muecke JS, Newland HS, Ryan P, Ramsay E, Aung M, Myint S, et al. Awareness of diabetic eye disease among general practitioners and diabetic patients in Yangon, Myanmar. Clin Exp Ophthalmol 2008 Apr;36(3):265-273. [doi: 10.1111/j.1442-9071.2008.01724.x] [Medline: 18412597$]$

12. James M, Turner DA, Broadbent DM, Vora J, Harding SP. Cost effectiveness analysis of screening for sight threatening diabetic eye disease. BMJ 2000 Jun 17;320(7250):1627-1631 [FREE Full text] [Medline: 10856062]

13. Tran K, Mendel TA, Holbrook KL, Yates PA. Construction of an inexpensive, hand-held fundus camera through modification of a consumer "point-and-shoot" camera. Invest Ophthalmol Vis Sci 2012 Nov 09;53(12):7600-7607 [FREE Full text] [doi: 10.1167/iovs.12-10449] [Medline: 23049089]

14. Banaee T, Ansari-Astaneh M, Pourreza H, Faal HF, Vatanparast M, Shoeibi N. Utility of $1 \%$ Tropicamide in Improving the Quality of Images for Tele-Screening of Diabetic Retinopathy in Patients with Dark Irides. Ophthalmic Epidemiol 2017;24(4):217-221 [FREE Full text]

15. Baeza M, Orozco-Beltrán D, Gil-Guillen VF, Pedrera V, Ribera MC, Pertusa S, et al. Screening for sight threatening diabetic retinopathy using non-mydriatic retinal camera in a primary care setting: to dilate or not to dilate? Int J Clin Pract 2009 Mar;63(3):433-438. [doi: 10.1111/j.1742-1241.2008.01921.x] [Medline: 19222628]

16. Aptel F, Denis P, Rouberol F, Thivolet C. Screening of diabetic retinopathy: effect of field number and mydriasis on sensitivity and specificity of digital fundus photography. Diabetes Metab 2008 Jun;34(3):290-293. [doi: 10.1016/j.diabet.2007.12.007] [Medline: 18406188 ]

17. Scanlon P, Malhotra R, Thomas G, Foy C, Kirkpatrick J, Lewis-Barned N, et al. The effectiveness of screening for diabetic retinopathy by digital imaging photography and technician ophthalmoscopy. Diabet Med 2003 Jun;20(6):467-474. [Medline: $\underline{12786681]}$

18. Lopez-Bastida J, Cabrera-Lopez F, Serrano-Aguilar P. Sensitivity and specificity of digital retinal imaging for screening diabetic retinopathy. Diabet Med 2007 Apr;24(4):403-407. [doi: 10.1111/j.1464-5491.2007.02074.x] [Medline: 17298591]

19. Maberley D, Cruess AF, Barile G, Slakter J. Digital photographic screening for diabetic retinopathy in the James Bay Cree. Ophthalmic Epidemiol 2002 Jul;9(3):169-178. [Medline: 12045884]

20. Murgatroyd H, Ellingford A, Cox A, Binnie M, Ellis JD, MacEwen CJ, et al. Effect of mydriasis and different field strategies on digital image screening of diabetic eye disease. Br J Ophthalmol 2004 Jul;88(7):920-924 [FREE Full text] [doi: 10.1136/bjo.2003.026385] [Medline: 15205238 ]

21. Al Sabti K, Raizada S, Wani VB, Al Ajmi M, Gayed I, Sugathan TN. Efficacy and reliability of fundus digital camera as a screening tool for diabetic retinopathy in Kuwait. J Diabetes Complications 2003;17(4):229-233. [Medline: 12810247]

22. Hansen AB, Hartvig NV, Jensen MS, Borch-Johnsen K, Lund-Andersen H, Larsen M. Diabetic retinopathy screening using digital non-mydriatic fundus photography and automated image analysis. Acta Ophthalmol Scand 2004 Dec;82(6):666-672 [FREE Full text] [doi: 10.1111/j.1600-0420.2004.00350.x] [Medline: 15606461]

23. UNDP. United Nations. 2015. UNDP's role and leadership on achieving the MDGs in Sri Lanka URL: http://www.lk.undp.org/ content/srilanka/en/home/post-2015/mdgoverview.html [accessed 2018-07-05] [WebCite Cache ID 70gZkAVim]

24. Katulanda P, Rathnapala D, Sheriff R, Matthews D. Province and ethnic specific prevalence of diabetes among Sri Lankan adults. Sri Lanka J Diabetes Endocrinol Metab 2012;1(1):2-7 [FREE Full text] 
25. Piyasena P, Murthy G. A situation analysis of diabetic eye care service delivery in health care institutions of the Western Province of Sri Lanka. Ceylon Med J 2017;62(3):205-206 [FREE Full text]

26. Whiting PF, Rutjes AWS, Westwood ME, Mallett S, Deeks JJ, Reitsma JB, et al. QUADAS-2: a revised tool for the quality assessment of diagnostic accuracy studies. Ann Intern Med 2011 Oct 18;155(8):529-536. [doi: 10.7326/0003-4819-155-8-201110180-00009] [Medline: 22007046]

27. Henricsson M, Karlsson C, Ekholm L, Kaikkonen P, Sellman A, Steffert E, et al. Colour slides or digital photography in diabetes screening--a comparison. Acta Ophthalmol Scand 2000 Apr;78(2):164-168 [FREE Full text] [Medline: 10794249]

28. Sundling V, Gulbrandsen P, Straand J. Sensitivity and specificity of Norwegian optometrists? evaluation of diabetic retinopathy in single-field retinal images - a cross-sectional experimental study. BMC Health Serv Res 2013;13(17) [FREE Full text]

29. Kuo H, Hsieh H, Liu R. Screening for diabetic retinopathy by one-field, non-mydriatic, 45 degrees digital photography is inadequate. Ophthalmologica 2005;219(5):292-296. [doi: 10.1159/000086114] [Medline: 16123556]

30. Early Treatment Diabetic Retinopathy Study Research Group. Grading diabetic retinopathy from stereoscopic color fundus photographs--an extension of the modified Airlie House classification. ETDRS report number 10.. Ophthalmology 1991 May;98(5 Suppl):786-806. [Medline: 2062513]

31. Perrier M, Boucher MC, Angioi K, Gresset JA, Olivier S. Comparison of two, three and four 45 degrees image fields obtained with the Topcon CRW6 nonmydriatic camera for screening for diabetic retinopathy. Can J Ophthalmol 2003 Dec;38(7):569-574. [Medline: 14740798]

32. Bhargava M, Cheung CY, Sabanayagam C, Kawasaki R, Harper CA, Lamoureux EL, et al. Accuracy of diabetic retinopathy screening by trained non-physician graders using non-mydriatic fundus camera. Singapore Med J 2012 Nov;53(11):715-719 [FREE Full text] [Medline: 23192497]

33. Owens DR, Gibbins RL, Lewis PA, Wall S, Allen JC, Morton R. Screening for diabetic retinopathy by general practitioners: ophthalmoscopy or retinal photography as $35 \mathrm{~mm}$ colour transparencies? Diabet Med 1998 Feb;15(2):170-175. [doi: 10.1002/(SICI)1096-9136(199802)15:2<170::AID-DIA518>3.0.CO;2-H] [Medline: 9507921]

34. Ruamviboonsuk P, Wongcumchang N, Surawongsin P, Panyawatananukul E, Tiensuwan M. Screening for diabetic retinopathy in rural area using single-field, digital fundus images. J Med Assoc Thai 2005 Feb;88(2):176-180. [Medline: 15962667]

35. Scanlon PH, Foy C, Malhotra R, Aldington SJ. The influence of age, duration of diabetes, cataract, and pupil size on image quality in digital photographic retinal screening. Diabetes Care 2005 Oct;28(10):2448-2453. [Medline: 16186278]

\author{
Abbreviations \\ DM: diabetes mellitus \\ DR: diabetic retinopathy \\ DRS: diabetic retinopathy screening \\ DRSP: diabetic retinopathy screening program \\ HIC: high-income country \\ LMICs: low-income and middle-income countries \\ NPDR: nonproliferative diabetic retinopathy
}

Edited by G Eysenbach, N Kuter; submitted 27.04.18; peer-reviewed by M Ameen, G Leontidis, G Lim, IJ Wang; comments to author
27.06.18; revised version received 05.07.18; accepted 13.09.18; published 10.12.18
Please cite as:
Piyasena MMPN, Gudlavalleti VSM, Gilbert C, Yip JLY, Peto T, MacLeod D, Fonseka C, Kulatunga A, Bandutilake BGWMKCB,
Dhanapala M, Pathirana L, Dissanayake H
Development and Validation of a Diabetic Retinopathy Screening Modality Using a Hand-Held Nonmydriatic Digital Retinal Camera
by Physician Graders at a Tertiary-Level Medical Clinic: Protocol for a Validation Study
JMIR Res Protoc 2018;7(12):e10900
URL: $\underline{\text { http://www.researchprotocols.org/2018/12/e10900/ }}$
doi: $10.2196 / 10900$
PMID: $\underline{30530458}$

CMapa Mudiyanselage Prabhath Nishantha Piyasena, Venkata S Murthy Gudlavalleti, Clare Gilbert, Jennifer LY Yip, Tunde Peto, David MacLeod, Charith Fonseka, Aruna Kulatunga, BGWMKCB Bandutilake, Mangala Dhanapala, Lalani Pathirana, Heshani Dissanayake. Originally published in JMIR Research Protocols (http://www.researchprotocols.org), 10.12.2018. This is an open-access article distributed under the terms of the Creative Commons Attribution License 
(https://creativecommons.org/licenses/by/4.0/), which permits unrestricted use, distribution, and reproduction in any medium, provided the original work, first published in JMIR Research Protocols, is properly cited. The complete bibliographic information, a link to the original publication on http://www.researchprotocols.org, as well as this copyright and license information must be included. 